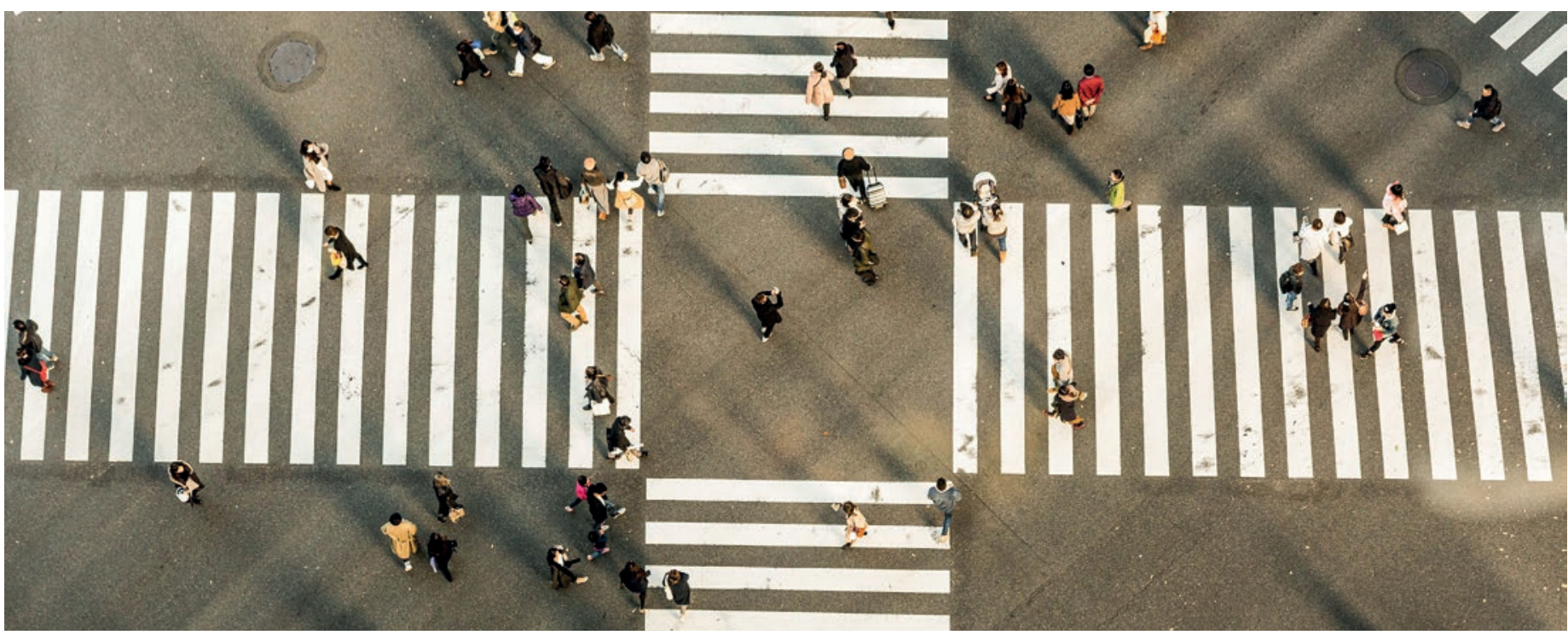

Entwicklung der Universitätsspitäler

\title{
Mehr als eine Machtfrage: Die Zukunft der universitären Medizin
}

\section{Christof Schmitz ${ }^{a}$ Peter Berchtold ${ }^{\mathrm{b}}$}

${ }^{a}$ college M, Bern; ${ }^{b}$ ISPM, Universität Bern, Bern

Die Konflikte und Krisen von Universitätsspitälern erregen mediale und politische Aufmerksamkeit. Die Diskussionen um das Universitätsspital Zürich (USZ) etwa verweisen symptomatisch auf ein generelles Problem im Bereich des Steuerungsregimes von Universitätsspitälern. Sie regen dazu an, über das Verhältnis von Medizin und Management und die Zukunft der universitären Medizin nachzudenken.

Im letzten Jahr herrschte viel medial notierte Unruhe rund um das USZ. Whistleblowings gaben zu reden, und mehrere Gutachten - insbesondere das der kantonsrätlichen Aufsichtskommission für Bildung und Gesundheit - sorgten für Aufmerksamkeit. Die dadurch angestossene öffentliche Diskussion fokussierte einerseits auf etwaige Fehler von Klinikdirektorinnen oder -direktoren und andererseits auf die Beziehung zwischen Spital und Universität. Oftmals verkürzte sie sich dabei auf eine Machtfrage und endete in einem Appell: Es gelte, die Macht der Direktion zu brechen, dann würde es im Spital wieder ruhig. Würde eine Hierarchie wie in anderen Unternehmen hergestellt, könne man (wieder) mit «ordentlichen» Verhältnissen rechnen.

\section{Kernproblem ist der Steuerungsbedarf}

So sehr Machtfragen hier relevant sind, so wenig wird dieser Fokus der Tragweite der Geschichte gerecht.
Einer Geschichte, die weit über Zürich und über unsere Landesgrenzen hinausreicht und die in einer doppelten Bewegung besteht: Einmal arbeiten die Universitätsspitäler intensiv daran, sich selbst besser steuern zu können. Sämtliche jüngeren Reorganisationen in den diversen Spitälern können in diese Richtung verstanden werden. Und diese Steuerungsthematik tangiert zwangsläufig das Verhältnis zwischen Medizin und Management, aber auch das Verhältnis zwischen Spital und Universität. Gleichzeitig hat sich die medizinische Forschung enorm dynamisiert. Die klassische Differenzierung von Grundlagenforschung und klinischer Forschung passt da wenig, weil sich die Forschung technologisiert, digitalisiert und interdisziplinär entwickelt hat.

Wenig verwunderlich daher, dass die klassische Schnittstelle zwischen Spital und Universität, die Personalunion von Klinikdirektorin oder Klinikdirektor mit Ordinaria bzw. Ordinarius, im Mittelpunkt dieser 
Auseinandersetzung steht. Handelt der Streit also vordergründig von (missbräuchlich verwendeter) Macht der Medizinalpersonen, dreht sich die eigentliche Auseinandersetzung darum, wie sich die Steuerung der Spitalorganisationen entwicklen wird, und damit um die Zukunft der universitären Medizin.

\section{Die Entwicklung der Spitäler}

Spitäler haben sich, entgegen einer immer wieder anzutreffenden Ansicht, in den letzten Jahrzehnten enorm gewandelt. Von Konglomeraten weniger, gering vernetzter Kliniken reformierten sie sich hin zu relativ selbständig steuernden Einheiten mit hochgradig vernetzten und komplexen Organisationen. Das ging zum einen mit rechtlicher Verselbständigung einher. Das

\section{Universitätsspitäler sind «anders» als andere Unternehmen. Sie müssen eine viel grössere Bandbreite differenter Perspektiven vernetzen.}

zeigt sich exemplarisch am USZ, das bis 2005 eine Abteilung der Zürcher Gesundheitsdirektion und schon damals eine Organisation mit rund 6000 Mitarbeitenden war. Heute ist das USZ öffentlich-rechtlich unabhängig, wenn auch immer noch an einer vergleichsweise kurzen Leine des Eigentümers. Mit grösserer Selbständigkeit und geändertem Vergütungssystem (DRG) stieg der Druck (und der Wunsch), sich als betriebswirtschaftliche Einheit besser steuern zu können, d.h., gezielter auf Entwicklungen reagieren und Strategien proaktiv entwickeln zu können. Dieser Wunsch nach intensivierter Steuerung stösst natürlich auf den Autonomiebedarf medizinisch-professioneller Entscheidungen. Die letzten 20 Jahre waren denn auch wesentlich vom Versuch geprägt, an dieser Schnittstelle sinnvolle Steuerungsregime einzurichten. Bereichsund Departementsbildungen, der Einzug von Klinikmanagement, die Professionalisierung von Budget- und Strategieprozessen etc. bieten Stichworte hierfür.

Zum anderen hat die Medizin ihr diagnostisches und therapeutisches Spektrum erheblich erweitert. Die Folge sind technologieträchtige, ressourcenaufwendige sowie hoch vernetzte Organisationsabläufe. Heute sind Universitätsspitäler ausserordentlich komplexe Gebilde von 40 bis 50 Kliniken und Instituten sowie vielfältigsten Berufsgruppen, hochgradig spezialisiert, intensiv vernetzt und weitreichend technologisiert. Etliche Kliniken haben heute den Umfang eines grösseren KMU und sind entsprechend anspruchsvoll zu organisieren, zu führen und zu managen. Die klassische Dreifach-Aufgabe, nämlich Klinik, Lehre und Forschung zu betreiben, wird um eine vierte, den Betrieb zu managen, ergänzt, und das alles unter Wirtschaftlichkeitszwängen und mit einer Fülle anspruchsvoller Stakeholder-Beziehungen.

\section{Perspektiven zusammenbringen}

Alle Universitätsspitäler, egal ob in Zürich, Genf oder anderswo in Europa, sind von dieser Anforderung gezeichnet, die übrigens jene anderer Unternehmen deutlich übersteigt. (Universitäts-)Spitäler sind, das kann man nicht genügend unterstreichen, «anders», nicht nur weil sie Expertenorganisationen sind, sondern weil sie eine viel grössere Bandbreite differenter Perspektiven zu vernetzen haben. Noch schärfer formuliert: Die verschiedenen Perspektiven (Klinik, Ökonomie, Forschung ...) passen grundsätzlich nicht zusammen. Sie bedürfen der fortlaufenden Übersetzung. Das eigentliche Thema lautet daher: Umgang mit «Perspektivendifferenz». Und das Problem dabei ist, das zeigt uns die moderne Soziologie, dass Perspektiven wie Wissenschaft, Medizin, Wirtschaft und Politik zueinander nicht in Hierarchien geordnet werden können. Um sich das schnell vor Augen zu führen: Keine Gesellschaft kann Interesse daran haben, dass ihre Medizin voll und ganz wirtschaftlichen Überlegungen folgen würde oder dass Wissenschaft vollständig politischem Willen unterworfen wäre.

\section{Die Machtfrage}

Die Universitätsspitäler stehen vor der Aufgabe, die Perspektivendifferenz von Klinik, Lehre, Forschung und Management in sinnvolle Steuerungsregime zu übersetzen. Sie müssen, wenn man so will, die nicht hierarchisch $\mathrm{zu}$ ordnenden Perspektiven in einen hierarchischen Zusammenhang bringen. Diese Aufgabe ist offensichtlich nicht frei von Paradoxien. Entsprechend ist die Vorstellung, das wäre alles einfach unter einen Hut zu bringen, nicht mehr als eine beliebte Mythenbildung. Deutlich formuliert: Weder eine starke Klinikdirektorin oder ein starker Klinikdirektor noch ein starker CEO bieten eine hinreichende Lösung. Im Wirtschaftsunternehmen reden wir auch nicht nur von der Geschäftsleiterin oder vom Geschäftsleiter, sondern sinnvollerweise von Geschäftsleitungen, also Gremien mit geteilten Führungsverantwortungen.

\section{Das Problem der Macht}

Die traditionelle organisatorische Lösung des Übersetzungsproblems von Klinik, Lehre und Forschung (und Management) war und ist die Personalunion der Klinik- 
direktorin oder des Klinikdirektors und der Ordinaria bzw. des Ordinarius. (Darüber hinaus bildet der Einsitz des Dekans oder der Dekanin in der Spitalleitung einen typischen Umgang mit dieser Differenz.) Diese Personalunion bedeutete, weil sie verschiedene Funktionen integrierte, immer mehr als eine Stelle. Wir sprechen von einem «Organisationsformat». Und diese Lösung hatte durchaus Raffinement.

Was auch immer die Lösungen sein werden, es wird über nichts weniger als über die Gestalt der zukünftigen universitären Medizin verhandelt.

Statt mühsam verhandeln zu müssen, wie Forschung und Klinik wechselseitig Zugang gewährt wird, bleibt es dieser Person überlassen zu entscheiden, welche Schwerpunkte und welche Projekte verfolgt und wie Ressourcen für Forschung und Klinik verteilt werden und - bezüglich Macht wichtig - wer welche Karriere machen würde. Für diese Leistung wurde Entscheidungsautonomie zugestanden. Und natürlich, solche Autonomie gibt Macht- wie auch Willkürpotenziale in die Hand. Der Preis des Formats war immer die hohe Abhängigkeit von Personen und die Gehorsamsbereitschaft, die daraus resultiert. Zum Vergleich: Anderen Organisationen ist es gänzlich fremd, in derartiger Dependenz von Personen agieren zu müssen.

Dieses altgediente und über lange Zeit erfolgreiche Organisationsformat gerät seit einiger Zeit unter Druck. Wichtigster Grund dafür sind die Akzentuierungen der Erwartungen, und dies von allen Seiten: Die Patientinnen und Patienten fordern, der Nachwuchs will anderes oder gar nicht mehr, das Controlling drückt, die Spitalleitung verlangt, die Forschung ist kompetitiv, und jeder dieser Bereiche findet sich zunehmend reguliert und vermessen. Der Ruf nach Neuerung ist enorm. Erfolgt kein rechtzeitiger Entwicklungsschritt und werden die Willkür- und Machtpotenziale in allzu blockierender Weise eingesetzt, suchen sich die damit verbundenen Konflikte andere Ventile, Whistleblowing zum Beispiel. Zürich «durfte» diesen Aspekt erleben.

\section{Quo vadis universitäre Medizin?}

Für die universitäre Medizin geht es im Kern um das Zusammenspiel zweier aufeinander angewiesener Institutionen, Spital und Universität, und die damit verbundenen Fragen der Gestaltung der Verhältnisse KlinikForschung und Klinik-Lehre. Dieses Thema erschöpft sich nicht in etwas protected time für die Forschung und vertraglich geklärten Zugängen zu Patientendaten, sondern zielt höher: Wie können (lokaler) Steuerungsbedarf und (globale) Forschungsdynamiken verknüpft werden?
Mit welchen Strukturen und welchen Stellen kann der Perspektivendifferenz begegnet werden?

Es reicht nicht, Personen zu suchen, die angesichts grosser Publikationslisten oder hoher Impact Factor versprechen, besonders gute Forschende zu sein, ebenso wenig wie es damit getan ist, jemanden zu suchen, der gut «führen» kann. Und es ist auch nicht damit getan, Doppelanstellungen abzuschaffen. Mit Verlaub, es ist schlichter Unsinn, die Perspektivendifferenz der universitären Medizin alleine mit noch so gutem Führungspersonal stemmen zu wollen. Bei mittlerweile stark akzentuierten Steuerungsbedürfnissen der Universitätsspitäler auf der einen und einer hoch dynamischen und extrem avancierten Forschung auf der anderen Seite reicht das nicht mehr hin. Geht es nicht mehr nur um Personen alleine, werden Strukturen und Strategien wichtig (in denen dann Personen wieder eine wichtige Rolle spielen können). Lernen kann man hier von anderen Ländern, etwa in der Gestaltung sogenannter clinical academic groups [1], die sowohl versorgungstechnisch wie akademisch interessant sind.

\section{Neudefinition des Organisationsformats}

Was auch immer zukünftig die Lösungen sein werden, indem über das Organisationsformat diskutiert wird, wird über die Gestalt einer zukünftigen universitären Medizin verhandelt. Nichts weniger. Wer das Organisationsformat ändert, greift unweigerlich in die Konfiguration der universitären Medizin ein und stellt die Frage, wie das Verhältnis von Klinik und Forschung (und Lehre und Management) gedacht und gestaltet werden soll. Und diese Frage ist für das Funktionieren eines Universitätsspitals wie einer medizinischen Fakultät von vitaler Bedeutung. Schliesslich gilt es hier, das Zusammenspiel einer breit gefächerten, technologisch fortschrittlichen, hoch interdisziplinären medizinischen Forschung mit klinischen Strukturen zu organisieren und clinical pull wie auch scientific push zu erzeugen.

\section{Vier Zukunftswünsche}

Es bleibt die Frage, was nun zu tun wäre und was es für mögliche Lösungsansätze gibt. Hierzu vier Wünsche für die Zukunft der universitären Medizin:

\section{In neuen Organisationsformen denken}

$\mathrm{Zu}$ wünschen wäre, erstens, dass weniger in Stellen und Personen denn in neuen Organisationsformen, -strukturen und -strategien gedacht werden würde. Ohnehin ist klar, dass grosse Kliniken nicht mehr durch Klinikdirektorinnen und Klinikdirektoren alleine zu führen sind. Bereits hier, noch ohne universitäre Anbindung, 
braucht es organisatorische Alternativen. Das Stichwort collective leadership soll an dieser Stelle genügen.

\section{Fächer differenzierter behandeln}

$\mathrm{Zu}$ wünschen wäre, zweitens, dass die mittlerweile so unterschiedlichen Fächer der Medizin differenzierter als bisher behandelt und nicht alle über den mittlerweile $\mathrm{zu}$ einfachen Leisten Klinikdirektorenstelle/ Lehrstuhl geschlagen würden. Was für die Rheumatologie sinnvoll sein kann, muss nicht für die Onkologie oder die Neurochirurgie gelten.

\section{Strategische Unklarheiten eingestehen}

Drittens und noch mehr zu wünschen wäre, dass man sich miteinander, Spital und Universität, eingestehen würde, welche immensen strategischen Unklarheiten in manchen ihrer Berufungen stecken. Um nur zwei Beispiele zu nennen: Wie soll die Zukunft einer akademischen viszeralen Chirurgie oder auch einer Allgemeinen Inneren Medizin aussehen? Wie soll diese Zukunft klinisch, wie soll sie akademisch prosperieren? Und wie passt das zusammen? Wie wäre das zu organisieren, damit alle Perspektiven erfolgreich integriert werden können? Wie erwähnt, eine Antwort auf diese Fragen erschöpft sich weder in Publikationsverzeichnissen noch Führungskompetenz-Bescheinigungen. Hier ist Struktur- und Strategiearbeit zu leisten. Gemeinsam. Wird

\section{Das Wichtigste in Kürze}

- Universitätsspitäler haben sich in den letzten Jahrzehnten zu hochkomplexen Unternehmen entwickelt. Zur Dreifach-Aufgabe von Klinik, Lehre und Forschung hat sich das Betriebsmanagement gesellt.

- Diese vier Aufgabenbereiche haben differente Perspektiven und Ansprüche, die nur schwer hierarchisiert werden können und eine grosse Übersetzungsleistung erfordern.

Dr. Christof Schmitz college M Haus der Akademien Laupenstrasse 7 CH-3001 Bern christof.schmitz[at] college-m.ch
- Die herkömmliche Lösung, die auf der Personalunion von Klinikdirektion und Ordinaria bzw. Ordinarius aufbaut, reicht deshalb nicht mehr aus. Es braucht grundlegende Strukturund Strategiereformen, um die Zukunft der universitären Medizin zu sichern. dieses Kapitalproblem einer unsicheren Zukunft über konkrete Rekrutierungsverfahren abgehandelt, muss man sich nicht wundern, wenn Konflikte ausbrechen.

\section{Tiefgreifende Lösungen suchen}

Und letztlich ist, viertens, von den politischen Kräften zu wünschen, dass sie von pseudo-einfachen Vorgaben absehen, die der eigentlichen Brisanz des Themas nicht gerecht werden, weil Universitätsspitäler die wertvollen, aber aussergewöhnlich komplexen Systeme sind, die sie nun mal sind.

Nicht zu vergessen ist dabei, dass ein guter und manchmal notwendiger Ausgangspunkt für neue Lösungen darin besteht, sich erst mal gemeinsam einzugestehen, dass es so wie bisher nicht weitergeht. Und dass die $\mathrm{Zu}$ kunft im Moment noch unklar ist. Das ist der Ursprung von Innovation und Experimenten, und die sind an dieser Stelle dringend vonnöten. Sonst droht der universitären Medizin bestenfalls Mittelmass.

\section{Bildnachweis}

Ryoji Iwata / Unsplash

\section{Literatur}

1 King's College London. Clinical academic groups [Internet]. https:// www.kcl.ac.uk/ioppn/depts/ps/about/cags/index (Zugriff am 30.6.2021)

\section{L'essentiel en bref}

- Les hôpitaux universitaires sont devenus des entreprises très complexes au cours des dernières décennies. A la triple mission d'hôpital, d'enseignement et de recherche s'est ajoutée la gestion opérationnelle.

- $\quad$ Ces quatre domaines de responsabilité ont des perspectives et des exigences différentes, difficiles à hiérarchiser et nécessitant beaucoup d'efforts de traduction.

- La solution conventionnelle basée sur l'union personnelle de la direction de la clinique et du professeur titulaire ne suffit donc plus. Des réformes structurelles et stratégiques fondamentales sont nécessaires pour assurer l'avenir de la médecine universitaire. 